10

\title{
Акустооптическая дифракция в парателлурите на медленной акустической моде. Повышение эффективности дифракции расходящегося света
}

\author{
(C) С.Н. Антонов, А.Л. Филатов
}

Институт радиотехники и электроники им. В.А. Котельникова РАН, 141190 Фрязино, Московская обл., Россия

e-mail: olga-ant@yandex.ru

\section{(Поступило в Редакцию 4 мая 2017 г.)}

Рассмотрены особенности брэгговской анизотропной акустооптической дифракции в парателлурите на медленной акустической волне. Показаны области частотно-угловых зависимостей, в которых существует эффективная дифракция, и сформулирована их практическая значимость. Получены численные значения для светового излучения с длиной волны света $1.06 \mu \mathrm{m}$. Предложен метод повышения эффективности дифракции расходящегося оптического излучения, что в свою очередь приводит к повышению быстродействия акустооптического модулятора. Метод основан на многочастотном электрическом управлении акустооптическим модулятором. Экспериментально показано, что в отличие от одночастотного управления достигается существенное подавление интенсивности нулевого порядка дифракции, с 30 до 8\%.

DOI: 10.21883/JTF.2018.06.46023.2323

\section{Введение}

АО (акустооптика) в практическом смысле - управление параметрами света, акустическими дифракционными решетками. Изучались разнообразные использования AO: анализаторы радиосигналов, приборы спектральной обработки изображений, оптические процессоры и др. „Ренессанс“ АО связан с революцией в лазерной технике - созданием мощных (более десятков $\mathrm{kW}$ ), компактных, с потрясающим КПД (вплоть до 50\%) волоконных лазеров. Принципиальные особенности АО управление очень интенсивным лазерным излучением $\left(\mathrm{kW} / \mathrm{cm}^{2}\right.$ и более), достаточно высокое быстродействие (вплоть до десятков ns), отсутствие механически перемещаемых элементов, малые вносимые световые потери (до единиц \%), практически неограниченный ресурс работы.

Работа посвящена АОМ (акустооптическим модуляторам) на базе оптически анизотропной среды. На практике основным материалом является монокристалл $\mathrm{TeO}_{2}$ в режиме дифракции на медленной акустической моде [1]. Основными параметрами АОМ являются быстродей-

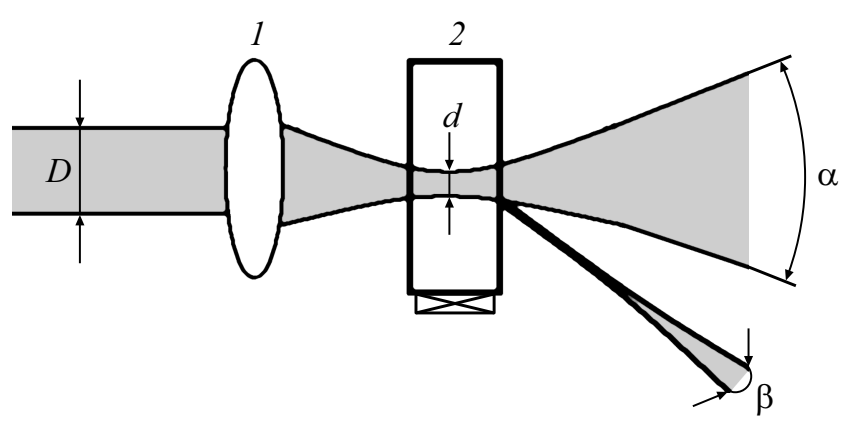

Рис. 1. Общая схема АОМ. ствие и эффективность. Широкое применение АОМ нашли в импульсных лазерах, для формирования „гигантского“ импульса. Модулятор устанавливается в полость резонатора, и работа ведется с нулевым порядком дифракции. При работающем модуляторе интенсивность нулевого порядка снижается до 5-15\%, лазер не излучает, а активная среда накапливает энергию возбуждения. При выключении АОМ добротность резонатора восстанавливается и происходит лазерная генерация.

На рис. 1 в общем виде приведена схема АОМ. Входной лазерный луч с апертурой $D$ фокусируется линзой 1 в АОМ 2 с перетяжкой диаметром $d$. На выходе модулятора существуют нулевой порядок с углом расходимости $\alpha$ и дифракционный с углом $\beta$. Практически $\beta$ всегда меньше $\alpha$, что в значительной мере и определяет эффективность модулятора. Быстродействие АОМ $\tau$ есть: $\tau=d / v$, где: $v-$ скорость звука в АО среде. Из последнего очевидно, что эффективность дифракции и быстродействие находятся в противоречии - чем меньше $d$, тем выше быстродействие, но и падает эффективность в силу увеличения $\alpha$.

Акцент исследований сделан на практически значимых режимах АО дифракции в парателлурите, связанных с повышением быстродействия АОМ.

\section{Виды геометрии анизотропной дифракции в парателлурите}

Векторная диаграмма рис. 2 иллюстрирует общий принцип анизотропной дифракции расходящегося света на одной частоте звука [2]. Здесь исходное излучение является необыкновенной модой с широким угловым спектром $I_{i}$. Дифракция, произошедшая на звуковой волне $\mathbf{k}_{s}$ со сменой моды (из необыкновенной в обыкновенную), 


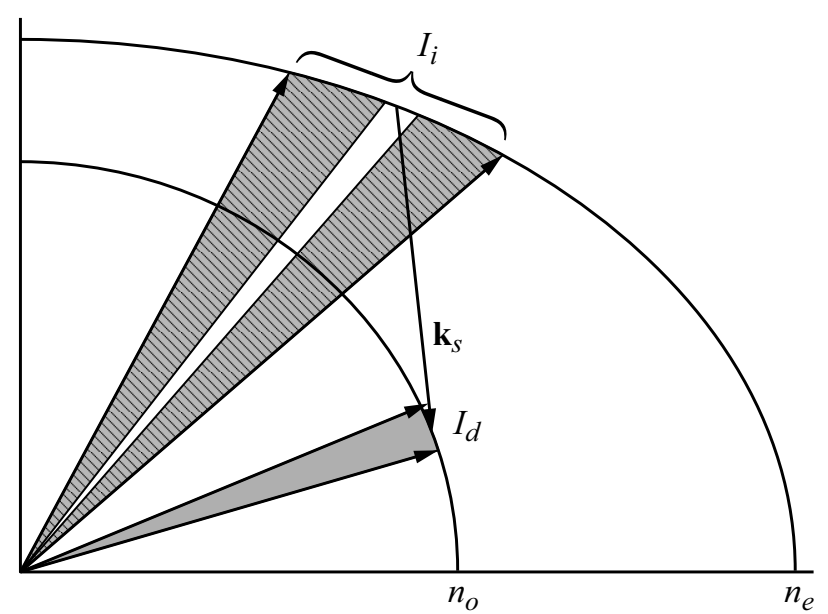

Рис. 2. Векторная диаграмма анизотропной дифракции расходящегося света.

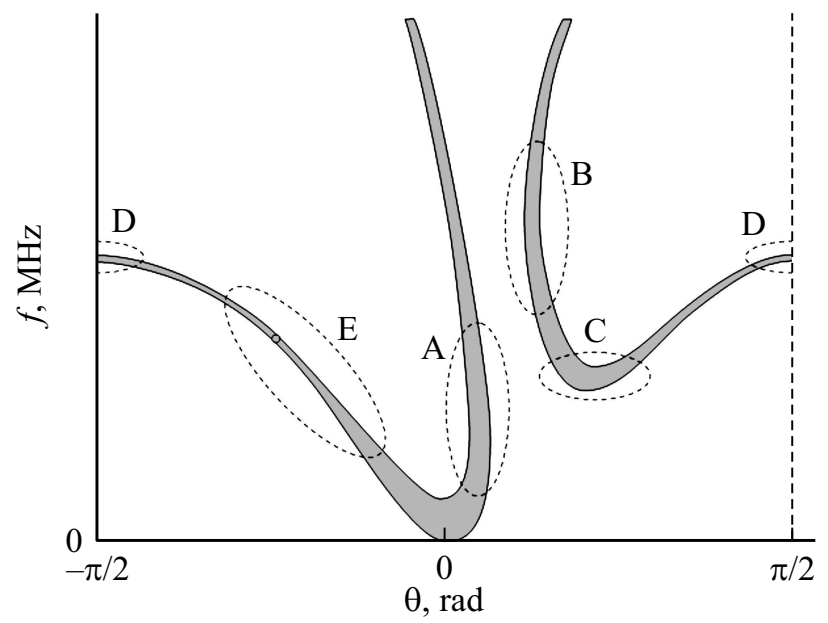

Рис. 3. Принципиальный вид частотно-угловых зависимостей анизотропной АО дифракции в парателлурите.

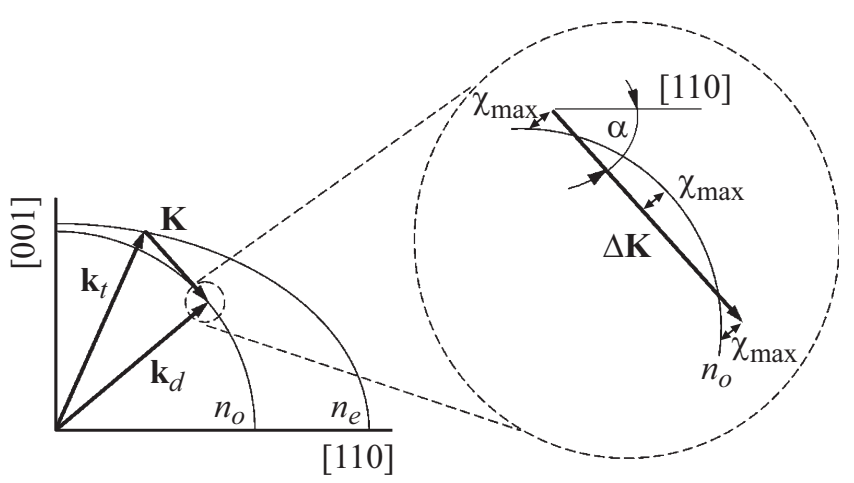

Рис. 4. Векторный вид параметра расстройки.

образует брэгговский порядок с угловым спектром $I_{d}$. В нашем примере угловой спектр дифрагированного света более узкий, чем спектр падающего света. Как следствие, дифрагирует лишь часть падающего на АОМ излучения.
В зависимости от взаимной ориентации волновых векторов света и звука можно выделить несколько специфических, различных по геометриям дифракции областей на частотно-угловых зависимостях, соответствующих применениям (АОМ, фильтры, дефлекторы и т. д.).

На рис. 3 приведен принципиальный вид частотно-угловой зависимости $\mathrm{AO}$ дифракции в парателлурите на медленной сдвиговой звуковой волне, волновой вектор которой лежит в плоскости (110) под углом нескольких градусов к направлению [110]. Здесь $\theta-$ угол в кристалле между направлением необыкновенной моды света и оптической осью, $f$ - частота звука. Закрашены области параметров, в которых происходит АО взаимодействие. Ширины областей иллюстрируют частотную полосу и угловую апертуру, в пределах которых происходит АО взаимодействие.

Области А и В соответствуют низкочастотному и высокочастотному анизотропным дефлекторам $[3,4]$. При такой геометрии взаимодействия для узких интервалов направлений падающего на кристалл света реализуется максимально возможная частотная полоса $\mathrm{AO}$ дифракции и, следовательно, большой угол сканирования. Область С соответствует геометрии оптического фильтра $[5,6]$, который характеризуется широкой угловой апертурой входного света, узкой частотной полосой, что определяет высокую селективность по длинам волн света. В области D имела бы место коллинеарная дифракция, если бы в ее центре не обращался в нуль фактор АО качества $M_{2}$. Тем не менее вблизи данной области возможна $\mathrm{AO}$ дифракция, применение которой связано с обработкой изображений [7]. Область Е характеризуется монотонной зависимостью $f$ от $\theta$, узкой частотной полосой акустических волн и малой угловой апертурой, в которой происходит АО дифракция.

Для численного сравнения параметров дифракции использовалась следующая приближенная зависимость показателей преломления обыкновенной и необыкновенной волн $n_{1}, n_{2}$ от угла $\theta$ :

$$
\left\{\begin{array}{l}
\frac{1}{n_{1}^{2}}=\frac{\sin ^{2} \theta}{n_{o}^{2}}+\frac{\cos ^{2} \theta}{n_{o}^{2}-n_{o} \Delta n}, \\
\frac{1}{n_{2}^{2}}=\frac{\sin ^{2} \theta}{n_{e}^{2}}+\frac{\cos ^{2} \theta}{n_{o}^{2}+n_{o} \Delta n} .
\end{array}\right.
$$

Здесь $n_{o}, n_{e}-$ главные показатели преломления обыкновенной и необыкновенной волн соответственно, $\Delta n-$ разность показателей преломления циркулярно поляризованных волн, распространяющихся вдоль оси кристалла, вызванная оптической активностью:

$$
\Delta n=\frac{\lambda \rho}{180^{\circ}},
$$

$\lambda$ - длина волны света, $\rho-$ удельное вращение плоскости поляризации. 

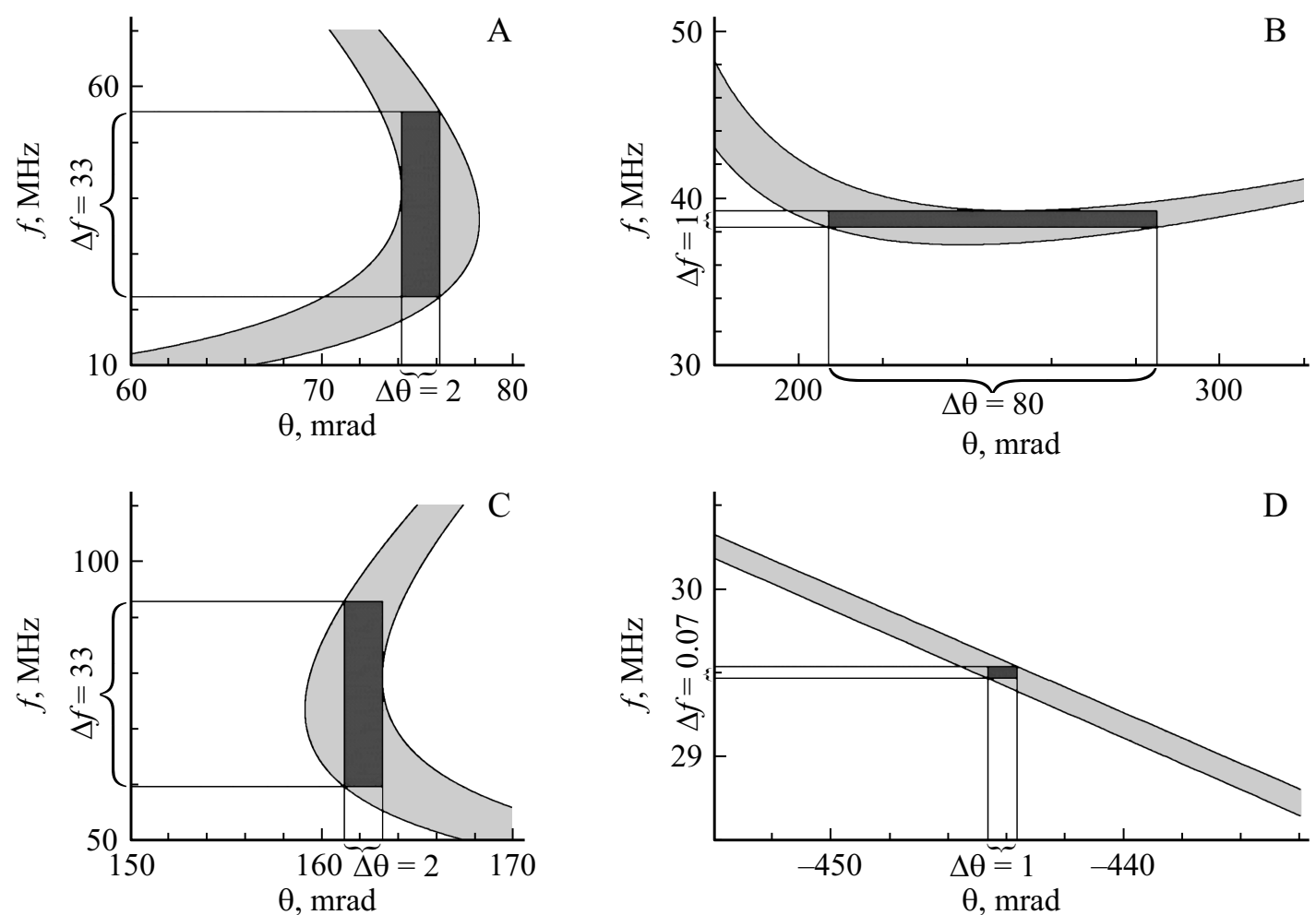

Рис. 5. Расчетные параметры частотно-угловых зависимостей для конкретных условий АО взаимодействия.

Предельная эффективность АО дифракции определяется величиной расстройки брэгговского синхронизма [8]:

$$
\chi=L\left|\mathbf{k}_{o}+\mathbf{k}_{s}-\mathbf{k}_{d}\right| .
$$

Здесь $\mathbf{k}_{o}, \mathbf{k}_{d}$ - волновые векторы взаимодействующих плосковолновых компонент исходного и дифрагированного света соответственно, $L$ - длина взаимодействия. Эффективности дифракции достигает 100\% при $\chi=0$ и $50 \%$ при $\chi=2.5$. Далее и в расчетах и при измерениях угловые и частотные диапазоны определяются по уровню предельной эффективности 50\%. Векторный вид параметра расстройки брэгговского синхронизма иллюстрирован на рис. 4. Здесь $\mathbf{K}-$ волновой вектор звука, $\mathbf{K}_{t}$ и $\mathbf{K}_{d}-$ падающего и дифрагированного света, соответственно $n_{o}, n_{e}$ - индикатрисы кристалла. $\Delta \mathbf{K}-$ изменение модуля вектора звука при перестройке его частоты.

На рис. 5 приведены результаты расчета для конкретного (экспериментального) случая: волновой вектор звука лежит в плоскости $(1 \overline{1} 0)$ под углом $6^{\circ}$ к оси [110], длина пьезопреобразователя $7 \mathrm{~mm}$, длина волны света $1.06 \mu \mathrm{m}$ (для большинства промышленных лазеров). Области (А, В, С, Е) те же, что и на рис. 3. Фигурными скобками показаны частотные и угловые диапазоны.

В наших экспериментах в качестве обоснования работоспособности метода будет использоваться режим дифракции А. Важно, что в этом случае сравнительно незначительные отклонения входного луча от центрального направления приводят к изменениям частоты звука, к большим углам отклонения. Более подробно об использовании этого свойства см. ниже.

\section{Метод и схема эксперимента}

Предлагаемый метод повышения быстродействия АОМ основан на следующем: обратимся к рис. 1 и 2. Очевидно, что при всех прочих условиях быстродействие тем выше, чем меньше размер перетяжки луча в полости АО кристалла. Однако уменьшение перетяжки

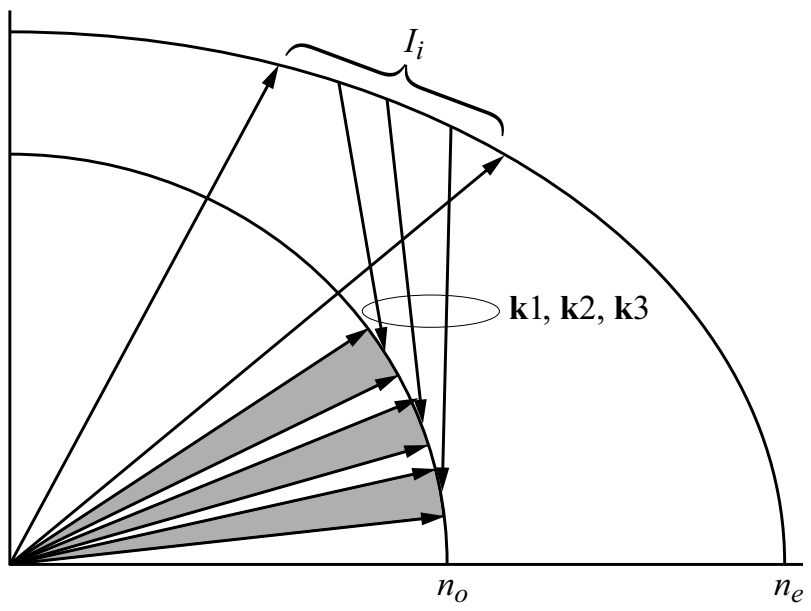

Рис. 6. Векторное пояснение дифракции на трех звуковых частотах. $\mathbf{k}_{1}, \mathbf{k}_{2}, \mathbf{k}_{3}-$ три вектора звуковых волн. 


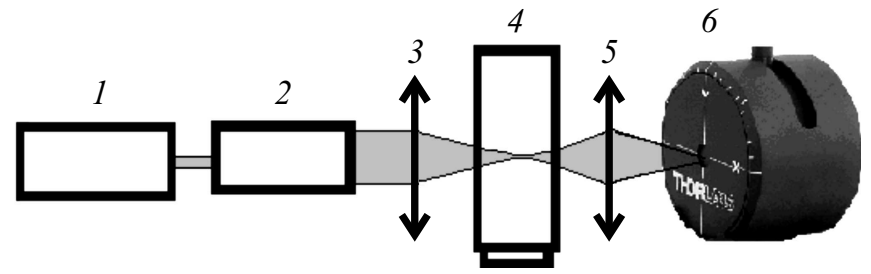

Рис. 7. Схема экспериментальной установки.

приводит к увеличению расходимости падающего на АОМ света. На одной фиксированной частоте ультразвука из широкого входного углового спектра в брэгговский порядок отклоняется лишь соответствующая узкая угловая часть, что и ограничивает эффективность.

Нами предложено при узкой перетяжке луча (значительной расходимости) осуществлять дифракцию одновременно из разных угловых частей падающего света, подавая на АОМ несколько (в частности, три) различных по частоте сигналов. Частоты звуковых волн должны быть подобраны таким образом, чтобы брэгговский синхронизм выполнялся из различных угловых частей падающего света. Это повышает суммарную эффективность дифракции расходящегося света. Рис. 6 поясняет метод.

На рис. 7 представлена схема экспериментальной установки. Излучение линейно-поляризованного одномодового лазера 1 с длиной волны $1.06 \mu \mathrm{m}$ направлялось на телескоп 2. На выходе телескопа коллимированное излучение фокусируется линзой 3 , образуя перетяжку в кристалле АОМ 4. Выходное излучение фокусируется линзой 5 на измеритель профиля оптического излучения 6. Использовался измеритель модели THORLABS BP104 с основными параметрами: скорость вращения щели - $10 \mathrm{~Hz}$, входное окно - $10 \mathrm{~mm}$, размер щели $10 \mu \mathrm{m}$. Оценочная относительная точность измерения положения луча $2-3 \%$.

Основные световые параметры: диаметр пятна на входе линзы 3 равен $1 \mathrm{~mm}$, диаметр перетяжки в полости АОМ $-100 \mu \mathrm{m}$. Исходя из этого предельное расчетное быстродействие модулятора составило $150 \mathrm{~ns}$. Геометрия АО взаимодействия соответствовала частотно-угловым зависимостям режима А рис. 5.

\section{Экспериментальные результаты}

Методика эксперимента заключалась в следующем. Входной свет был направлен на АОЯ под углом Брэгга для центра его углового распределения. Серия рис. 8 представляет пространственное распределение света на измерителе профиля и пересчитанное угловое на выходе АОМ по уровню $1 / e^{2}$. При всех измерениях амплитуда управляющего радиосигнала устанавливалась по критерию максимальной эффективности дифракции. На рис. 8, $а$ представлено распределение нулевого порядка дифракции без подачи сигнала на АОМ - исходное излучение с расходимостью $10 \mathrm{mrad}$. Здесь и далее $I-$ относительная эффективность. На рис. 8, $b$ график $1-$ вид распределения нулевого порядка дифракции при подаче одночастотного сигнала управления $41.5 \mathrm{MHz}$,
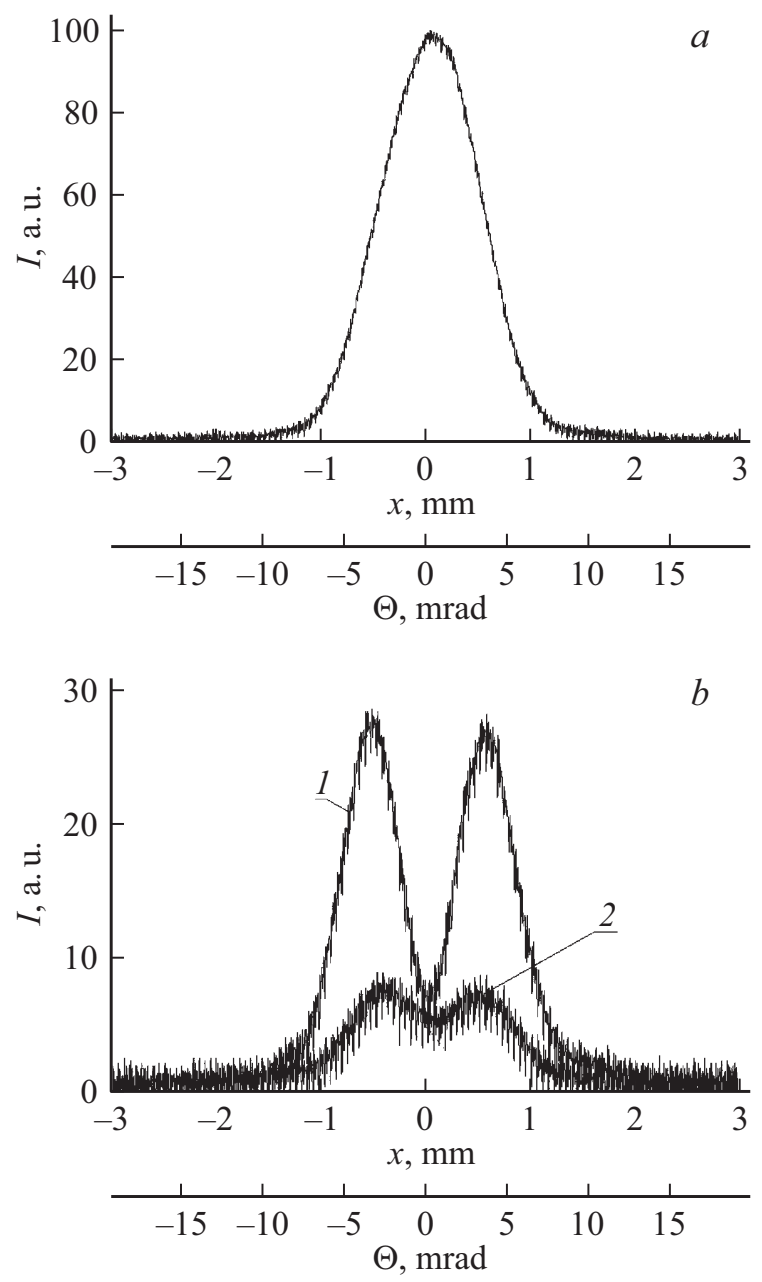

Рис. 8. Вид распределения светового излучения.

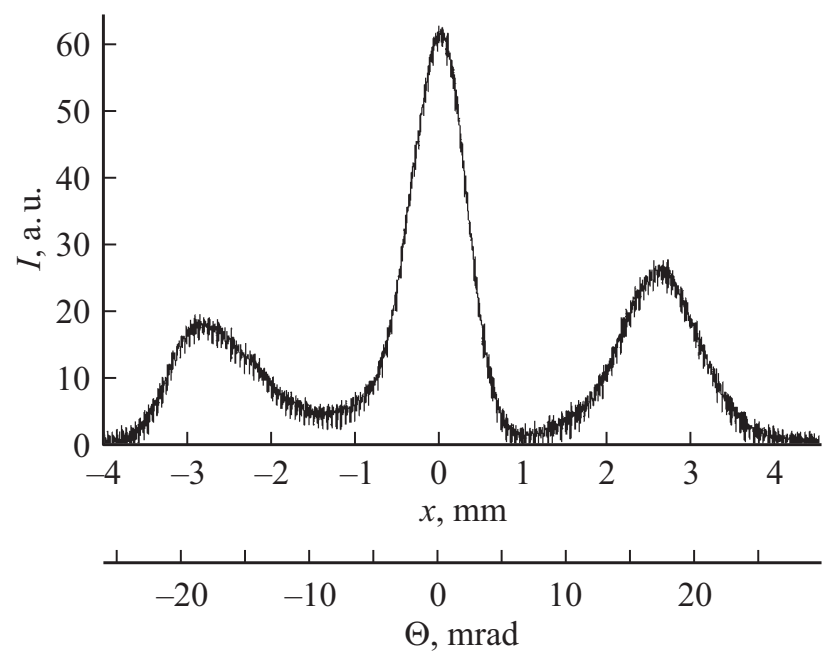

Рис. 9. Распределение интенсивности света в дифракционном порядке при подаче трех частот управлении. 
график 2 - подача трех частот управляющего сигнала: центральная $41.5 \mathrm{MHz}$ и две боковые 37.3 и $45.7 \mathrm{MHz}$.

Из рис. $8, b$ видно, что на одночастотном сигнале (график 1) из углового спектра падающего луча вырезается только центральная часть, и интенсивность нулевого порядка составила $30 \%$ от исходного. При трехчастотном сигнале (график 2) интенсивность нулевого порядка значительно снижается и составляет около $8 \%$. Отметим, что при трехчастотном сигнале эффективность подавления центра углового распределения примерно равна одночастотному случаю, однако боковые компоненты претерпевают существенное подавление.

На рис. 9 представлено световое поле дифракционного порядка при трехчастотном управлении в соответствии с рис. $8, b$. Масштаб по оси $x$ увеличен по сравнению с рис. 8. Из рис. 9 видно, что дифракционное поле состоит из трех неперекрывающихся лучей. Боковые лучи отстоят от центрального на угол около $18 \mathrm{mrad}$. Суть этой дифракции в том, что центральный луч образован отклонением из центральной угловой части падающего луча, а два других - из боковых частей луча. То, что эти лучи не перекрываются, есть свойство режима А, рис. 5, когда незначительное отклонение направления падающего луча требует большой перестройки частоты управляющего сигнала. Как следствие, разделение дифракционных лучей обеспечивает отсутствие интермодуляционных эффектов и существенное снижение интенсивности нулевого порядка дифракции. Экспериментальные данные находятся в удовлетворительном согласии с расчетом рис. 5, график $A$.

Таким образом, установлено и показано, что многочастотное управление АОМ позволяет повысить эффективность дифракции расходящегося лазерного излучения. Это, в свою очередь, приводит к повышению быстродействия. В экспериментальной ситуации быстродействие составило $150 \mathrm{~ns}$.

Отметим, что по сравнению с одночастотным режимом требуется увеличить суммарную подводимую к АОМ электрическую мощность в три раза. Однако это не будет приводить к нежелательным тепловым эффектам, если АОМ, как и лазер, будет работать в импульсном режиме (скважность на практике не менее 10).

Существенно, что метод в зависимости от задачи может быть применен и для других областей дифракции.
2. Предложен метод повышения эффективности дифракции расходящегося оптического излучения. Метод основан на использовании многочастотного управляющего электрического сигнала. При этом различные частотные компоненты электрического сигнала осуществляют дифракцию из различных угловых участков светового спектра.

3. На примере анизотропной дифракции в парателлурите показано, что в отличие от одночастотного управления трехчастотное значительно снижает интенсивность нулевого порядка дифракции. Для экспериментальной ситуации это снижение составило с 30 до $8 \%$.

Выражаем признательность к.ф.-м.н. А.В. Вайнеру за помощь в расчетах.

Работа выполнена по госзаказу ФИРЭ РАН.

\section{Список литературы}

[1] Антонов С.Н. // ЖТФ. 2016. Т. 86. Вып. 1. С. 132-135.

[2] Антонов С.Н., Вайнер А.В., Проклов В.В., Янгиров И.Ф. // Радиотехника и электроника. 2011. Т. 56. Вып. 7. С. 889895.

[3] Семенков В.П. // ЖТФ. 1981. Т. 51. Вып. 10. С. 2090.

[4] Антонов С.Н. // ЖТФ. 2016. Т. 86. Вып. 1. С. 136-139.

[5] Voloshinov V.B. // Opt. Eng. 1992. Vol. 31. N 10. P. 2089-2094.

[6] Voloshinov V.B., Molchanov V.Ya., Mosquera J.C. // Opt. Laser Technol. 1996. Vol. 28. N 2. P. 119-127.

[7] Voloshinov V.B., Yukhnevich T.V. // Appl. Opt. 2013. Vol. 52. P. 5912-5919.

[8] Корпел А. Акустооптика. М.: Мир, 1993. 240 с.

\section{Выводы}

1. Рассмотрен общий принципиальный вид частотно-угловых зависимостей АО дифракции в парателлурите на медленной сдвиговой звуковой волне, волновой вектор которой лежит в плоскости (11̄0) под углом нескольких градусов к направлению [110]. Рассчитаны конкретные параметры частотно-угловых зависимостей AО дифракции в парателлурите для длины волны света $1.06 \mu \mathrm{m}$. 